\title{
Complications of the surgical excision of encapsulated versus nonencapsulated lipomas: A retrospective analysis
}

\section{Won Hyuck Do, Young Woong Choi}

Department of Plastic and Reconstructive Surgery, Sanggye Paik Hospital, Inje University College of Medicine, Seoul, Korea
Background Lipomas are common benign soft tissue tumors composed of mature white adipocytes, with histological features including a well-circumscribed and lobular mass covered with a thin fibrous capsule. However, lipomas that are poorly demarcated from the surrounding fat are often encountered during surgery despite a postoperative histological diagnosis. We investigated the complications associated with different types of lipomas.

Methods This retrospective study included 119 patients who underwent lipoma excision and computed tomography (CT) imaging at our clinic between January 2011 and August 2018. We classified the lipomas as encapsulated or nonencapsulated according to the histology, CT findings, and clinical criteria. Nonencapsulated lipomas were defined as relatively heterogeneous without a distinct capsule, whereas encapsulated lipomas were homogeneous with a distinct capsule. The analyzed complications included delayed wound healing, which can cause prominent scarring, hematoma or seroma, and recurrence.

Results Encapsulated and nonencapsulated lipomas were diagnosed in $89(74.8 \%)$ and $30(25.2 \%)$ patients, respectively. Encapsulated lipomas occurred most commonly on the head, whereas nonencapsulated lipomas occurred most commonly on the neck and trunk $(P=0.000, P=0.002$, and $P=0.031$, respectively). The Fisher exact test showed a significantly higher incidence of delayed wound healing for nonencapsulated than encapsulated lipomas $(P=0.014)$.

Conclusions Preoperative classification of lipomas using CT imaging is important for predicting the incidence of postoperative complications. Direct excision is adequate for removing encapsulated lipomas. However, nonencapsulated lipomas might require alternative methods, such as ultrasonic liposuction, to prevent postoperative complications. Our results will help reduce the incidence of scarring by providing guidance on surgical methods.

Keywords Lipoma / Excision / Complications
Received: Apr 1, 2019 Revised: Jul 4, 2019 Accepted: Jul 9, 2019 Correspondence: Young Woong Choi Department of Plastic and Reconstructive Surgery, Sanggye Paik Hospital, Inje University College of Medicine, 1342 Dongil-ro, Nowon-gu, Seoul 01757, Korea

Tel: +82-2-950-1048, Fax: +82-2-932-6373, E-mail: pshero2@naver.com

Copyright @ 2019 The Korean Society for Aesthetic Plastic Surgery.

This is an Open Access article distributed under the terms of the Creative Commons Attribution Non-Commercial License (http://creativecommons.org/licenses/by-nc/4.0/) which permits unrestricted non-commercial use, distribution, and reproduction in any medium, provided the original work is properly cited. www.e-aaps.org

\section{INTRODUCTION}

Lipomas are one of the most common mesenchymal tumors, and can develop in any fat-containing region of the body. They are typically located subcutaneously and consist of mature fatty tissue [1]. Lipomas on the trunk and limbs rarely present a diagnostic problem, and surgical excision is the mainstay of management [2].

Direct excision of the lipoma is the standard approach for surgery. However, direct excision may leave scars in cosmetically un- 
desirable locations and patients who are susceptible to scarring are often disinclined to receive treatment using this method. Concerns regarding cosmesis have prompted several investigators to approach lipomas in a less invasive manner, including the use of remote incisions with endoscopic assistance for the excision of lipomas [3]. Patient satisfaction is very high with less invasive approaches, especially in cases where an open approach with an incision over the lipoma was expected [4]. However, techniques involving remote incisions can cause complications such as flap necrosis, wound dehiscence, and burns.

Generally, lipomas present as slow-growing, soft, and bulging masses. The histological features of lipoma include a well-circumscribed and lobular mass covered with a thin fibrous capsule $[5,6]$. However, lipomas that are not demarcated from the surrounding fat are often encountered during surgery despite a postoperative histological diagnosis [7]. Roberts et al. [8] described cases of unclassifiable lipomas composed of palpable fatty masses without a definitive capsule, identified in retrospective reviews of magnetic resonance images. They defined lipomas without a definitive capsule as nonencapsulated lipomas and those with a definitive capsule as encapsulated lipomas. In this study, we investigated the incidence of complications for both types of lipomas.

\section{METHODS}

This retrospective study included 119 patients with a pathological

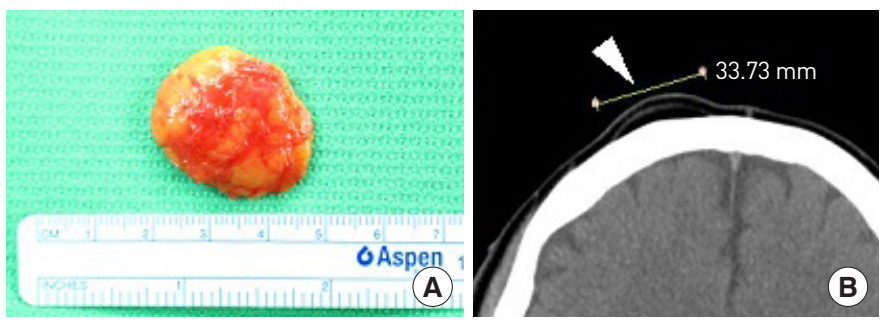

Fig. 1. Encapsulated lipoma. (A) Intraoperative photograph of an encapsulated lipoma with a capsule. (B) Computed tomography imaging of an encapsulated lipoma surrounded by a capsule (arrowhead).
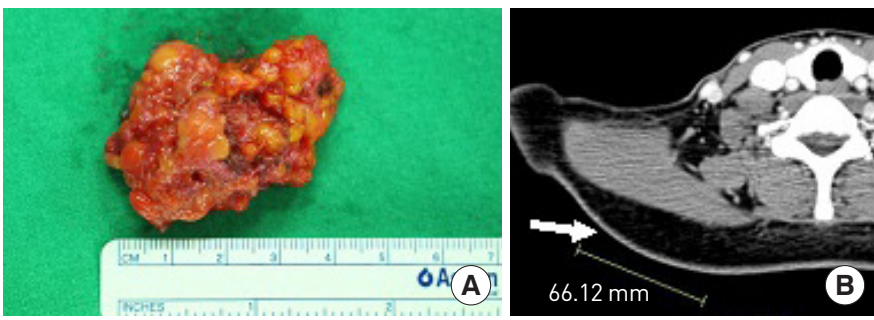

Fig. 2. Nonencapsulated lipoma. (A) Intraoperative photograph of a nonencapsulated lipoma without a capsule. (B) Computed tomography imaging of a nonencapsulated lipoma, which is seen as a bulging contour in the posterior aspect of the right shoulder (arrow). diagnosis of lipoma who underwent lipoma excision and computed tomography (CT) imaging at Sanggye Paik Hospital during a 7-year period between January 2011 and August 2018. Patients who had lipomatosis or other lipoma subtypes such as hibernoma, fibrolipoma, angiolipoma, myelolipoma, or spindle cell lipoma were excluded to ensure that the analysis was unbiased. We reviewed the patients' medical records, photographs of the wounds, specimens, and CT imaging studies to determine the classification of the lipoma and to evaluate the rate of complications. Informed consent was obtained from all patients included in this retrospective study of their medical records and CT imaging. A consensus panel that included a professor of plastic surgery and a junior resident in plastic surgery reviewed the imaging studies in a random order.

If more than $25 \%$ of the circumference of the lipoma was encapsulated in at least one plane with a smooth, linear margin, as specified by Roberts et al. [8], the mass was defined as an "encapsulated lipoma." A mass was considered to be nonencapsulated if it had less than $25 \%$ of its circumference delineated by a linear margin in all planes by the same method (Figs. 1,2).

The clinical characteristics of the patients, including age, sex, lipoma location, size, and postoperative complications, were recorded. The complications analyzed in this study were delayed wound healing (healing more than 14 days after surgery), recurrence, seroma, and hematoma. Results are presented as the mean \pm standard deviation or as number and percentage. The Pearson chi-square test and the Fisher exact test were used to compare the clinical and

Table 1. Patient demographics and outcomes

\begin{tabular}{|c|c|c|c|}
\hline Variable & $\begin{array}{l}\text { Encapsulated } \\
\text { lipoma }(n=89)\end{array}$ & $\begin{array}{l}\text { Nonencapsulated } \\
\text { lipoma }(n=30)\end{array}$ & $P$-value \\
\hline Age (yr) & $44.04 \pm 13.37$ & $46.03 \pm 16.83$ & 0.250 \\
\hline Range & $12-85$ & $2-74$ & \\
\hline Sex & & & 0.210 \\
\hline Male & $45(50.5)$ & $11(36.6)$ & \\
\hline Female & $44(49.4)$ & 19 (63.3) & \\
\hline $\begin{array}{l}\text { Greatest resected tumor } \\
\text { length }(\mathrm{cm})\end{array}$ & $2.37 \pm 1.43$ & $4.08 \pm 2.66$ & 0.128 \\
\hline \multicolumn{4}{|l|}{ Location } \\
\hline Head & 53 (59.5) & $2(6.6)$ & 0.000 \\
\hline Neck & $6(6.7)$ & $9(30.0)$ & 0.002 \\
\hline Trunk & 19 (21.3) & $13(43.3)$ & 0.031 \\
\hline Upper extremity & $10(11.2)$ & $5(16.6)$ & 0.525 \\
\hline Lower extremity & $1(1.1)$ & $1(3.3)$ & 0.442 \\
\hline \multicolumn{4}{|l|}{ Complications } \\
\hline Delayed healing & $1(14.2)$ & $4(66.6)$ & 0.014 \\
\hline Seroma, hematoma & $6(85.7)$ & $1(16.6)$ & 0.678 \\
\hline Recurrence & 0 & $1(16.6)$ & 0.252 \\
\hline
\end{tabular}

Values are presented as mean \pm SD or number $(\%)$.

$P$-values $<0.05$ were considered to indicate statistical significance. 
radiological characteristics between the two lipoma groups. P-values less than 0.05 were considered to indicate statistical significance. Statistical analyses were performed using SPSS version 22.0 (IBM Corp., Armonk, NY, USA).

\section{RESULTS}

Table 1 presents the demographic characteristics of the participants (including age, sex, size, lipoma location, and complications). The 119 lipomas for which CT imaging findings were available were categorized as 89 encapsulated lipomas $(74.8 \%)$ and 30 nonencapsulated lipomas (25.2\%).

The study population was $47 \%$ male (56/119). The Fisher exact test showed that the greatest resected tumor length was not significantly different between the nonencapsulated lipoma group and to the encapsulated lipoma group (2.37 cm vs. $4.08 \mathrm{~cm}, \mathrm{P}=0.128)$. Clinically, there were no significant differences in patients' age, sex, and greatest resected tumor length between the encapsulated lipoma group and nonencapsulated lipoma group.

In this study, we classified the location of the lipomas into five different anatomical locations. The head was the most frequently involved location for encapsulated lipomas, whereas the neck and trunk were frequent sites of nonencapsulated lipomas $(\mathrm{P}=0.000$, $\mathrm{P}=0.002$, and $\mathrm{P}=0.031$ ). There was no significant difference between the upper and lower extremities regarding the presence of a lipoma capsule. This study showed that nonencapsulated lipomas had a significantly higher incidence of delayed wound healing than encapsulated lipomas $(\mathrm{P}=0.014)$. In patients who experienced delayed healing, healing by secondary intention with a continuous simple dressing was performed. Complete healing was achieved in 26.4 days on average.

The rates of seroma or hematoma formation and recurrence showed no statistically significant differences between the groups. Hematoma and seroma were treated with continuous aspiration and compressive dressing in five cases, and stitch-out, old blood clot removal, and re-suturing in two cases. All patients healed without further complications after the procedure described above.

\section{DISCUSSION}

Lipomas are the most common benign mesenchymal neoplasm in soft tissues, with a prevalence of 2.1 per 1,000 people [9]. Lipomas typically present as soft, solitary, painless, subcutaneous nodules that are mobile and not associated with epidermal changes. As lipomas rarely turn malignant and tend to be asymptomatic, the importance of lipoma classification is often overlooked. Before surgery, lipomas are often simply diagnosed by inspection and palpation, and at most, ultrasonography may be performed.

Ultrasonography of subcutaneous tumors is useful for obtaining information about the nature, depth, size, and adjacent vascular structures in some cases [10]. In the past, ultrasonography was the first choice of imaging modality because of its usefulness and costeffectiveness compared to other imaging techniques [11]. However, the sonographic appearances of lipomas, and in particular their echogenicity, has been shown to be variable depending on the degree of connective tissue and other reflective interfaces present within a lipoma $[12,13]$. Classification of lipomas by ultrasonography was not considered a suitable method for this study because even radiologists have shown discordance in the categorization of lipomas [14]. In addition, since insurance covers CT scans for lipoma diagnosis, CT imaging is considered to be more helpful than ultrasonography for accurately determining the characteristics of lipomas.

Before surgery, encapsulated lipomas and nonencapsulated lipomas could not be differentiated; however, their characteristics were distinguished on CT scans. Grossly, the specimens different in terms of the presence of a whitish capsule and the size of each lobule. Histologically, the size and form of adipocytes were similar when evaluated by hematoxylin and eosin staining (Fig. 3). Chang et al. [7] suggested that nonencapsulated lipomas appeared to contain
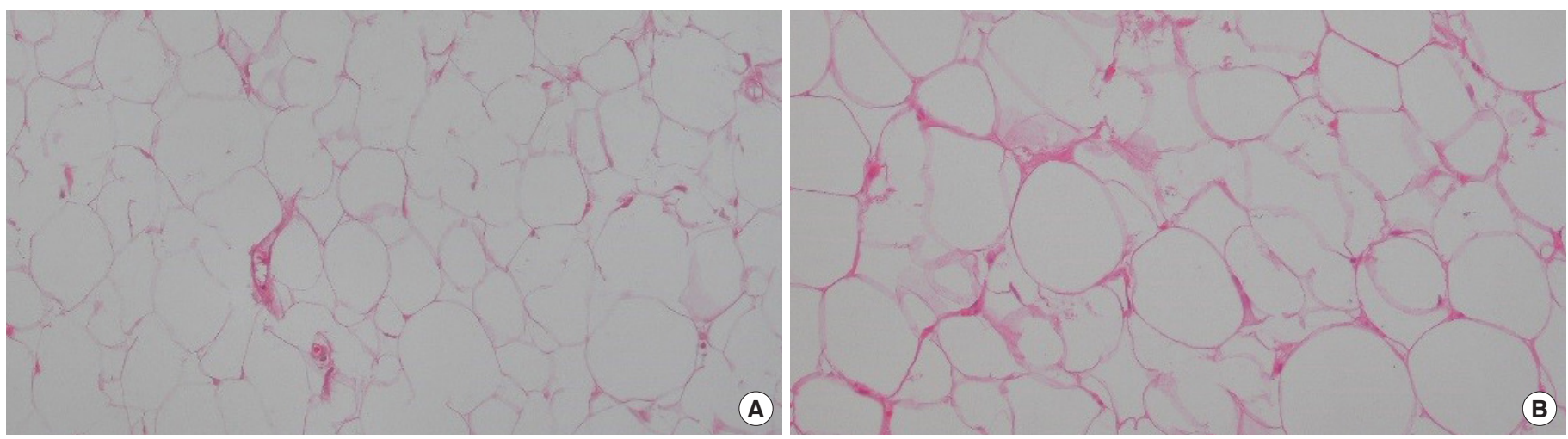

Fig. 3. Histological findings of lipomas. The adipocytes of (A) an encapsulated lipoma and (B) a nonencapsulated lipoma show no significantly different characteristics, such as size or form $(H \& E, \times 200)$. 
thick fibrous septa, instead of a circumferential capsule in encapsulated lipomas.

According to this study, there was a significant difference in the risk of complications after lipoma resection depending on the presence of a capsule. Firstly, the authors concluded that in an encapsulated lipoma, the margins with the surrounding soft tissue are clear; therefore, capsule-containing resection is possible. However, in a nonencapsulated lipoma, it is difficult to define the plane of resection, which can increase the likelihood of delayed wound healing.

Secondly, nonencapsulated lipomas are more likely than encapsulated lipomas to develop in a dependent position. In this study, nine nonencapsulated lipomas occurred in the posterior aspect of the neck and 12 nonencapsulated lipomas occurred in the back, which was significantly higher than the incidence of encapsulated lipomas in those areas. If patients spend a long time lying down, with continuous irregular application of pressure over the site, the resultant poor circulation could aggravate the wound healing process.

Instead of surgical excision, liposuction could be an alternative method of removal for nonencapsulated lipomas. Al-basti and ElKhatib [15] suggested that liposuction, with a minimally invasive technique, is an excellent alternative to surgical excision in lipomas, especially in cases of moderate to large lipomas or multiple lipomatosis.

This study has several limitations. First, because of the retrospective characteristics of the study, selection bias could not be eliminated. Patients who did not undergo CT imaging for various reasons, such as high cost or resistance to radiation exposure, were excluded from this study. Additionally, patients who did not receive surgical treatment were excluded.

Secondly, since past records were accessed, accurate information on complications and the presence or absence of drainage was sometimes not present. Seroma or hematoma formation is strongly influenced by the presence or absence of drainage; hence, some inaccuracies in the data were possible.

In conclusion, when comparing the incidence of postoperative complications, it is important to preoperatively classify the type of lipoma using CT imaging. Direct excision is an adequate method for the removal of encapsulated lipomas, but alternative methods would be better for nonencapsulated lipomas to prevent postoperative complications. The results of this study will help reduce the incidence of scarring by providing preoperative guidance regarding the choice of surgical method.

\section{NOTES}

\section{Conflict of interest}

No potential conflict of interest relevant to this article was reported.

\section{Ethical approval}

The study was performed in accordance with the principles of the Declaration of Helsinki.

\section{Patient consent}

The patients provided written informed consent for the publication and the use of their images.

\section{ORCID}

Won Hyuck Do

Young Woong Choi

\author{
https://orcid.org/0000-0003-1114-2488
}

https://orcid.org/0000-0002-2211-2981

\section{REFERENCES}

1. McTighe S, Chernev I. Intramuscular lipoma: a review of the literature. Orthop Rev (Pavia) 2014;6:5618.

2. Brass D, Oliphant TJ, McHanwell S, et al. Successful treatment of forehead lipoma depends on knowledge of the surgical anatomy: a stepby-step guide. Clin Exp Dermatol 2016;41:3-7.

3. Funayama E, Minakawa H, Oyama A. Forehead lipoma resection via a small remote incision using a surgical raspatory. J Am Acad Dermatol 2007;56:458-9.

4. Pereira JA, Schonauer F. Lipoma extraction via small remote incisions. Br J Plast Surg 2001;54:25-7.

5. Dalal KM, Antonescu CR, Singer S. Diagnosis and management of lipomatous tumors. J Surg Oncol 2008;97:298-313.

6. Signorini M, Campiglio GL. Posttraumatic lipomas: where do they really come from? Plast Reconstr Surg 1998;101:699-705.

7. Chang H, Park SO, Jin US, et al. Characterization of two distinct lipomas: a comparative analysis from surgical perspective. J Plast Surg Hand Surg 2018;52:178-84.

8. Roberts CC, Liu PT, Colby TV. Encapsulated versus nonencapsulated superficial fatty masses: a proposed MR imaging classification. AJR Am J Roentgenol 2003;180:1419-22.

9. Kumari KL, Babu VP, Kumar SV. Giant intermuscular lipoma of neck and chest: a case report with review of literature. Int J Res Med Sci 2015; 3:1521-3.

10. Kuwano Y, Ishizaki K, Watanabe R, et al. Efficacy of diagnostic ultrasonography of lipomas, epidermal cysts, and ganglions. Arch Dermatol 2009;145:761-4.

11. Bloem JL. Imaging of soft tissue tumors. J Belge Radiol 1992;75:26573.

12. Lin J, Jacobson JA, Fessell DP, et al. An illustrated tutorial of musculoskeletal sonography: part 4, musculoskeletal masses, sonographically guided interventions, and miscellaneous topics. AJR Am J Roentgenol 2000;175:1711-9.

13. Wild JJ, Reid JM. Further pilot echographic studies on the histologic structure of tumors of the living intact human breast. Am J Pathol 1952;28:839-61.

14. Inampudi P, Jacobson JA, Fessell DP, et al. Soft-tissue lipomas: accura- 
cy of sonography in diagnosis with pathologic correlation. Radiology 2004;233:763-7.

15. Al-basti HA, El-Khatib HA. The use of suction-assisted surgical ex- traction of moderate and large lipomas: long-term follow-up. Aesthetic Plast Surg 2002;26:114-7. 